\title{
Mean Effective Gain of Compact WLAN Genetic Printed Dipole Antennas in Indoor-Outdoor Scenarios
}

\author{
Pedro Luis Carro and Jesus de Mingo \\ University of Zaragoza,Electronic Engineering and Communications Department, \\ Zaragoza 50018, Spain \\ plcarro@unizar.es, mingo@unizar.es \\ http://diec.unizar.es
}

\begin{abstract}
Two dual-printed dipole antennas for WLAN applications operating in the $802.11 \mathrm{a} / \mathrm{b} / \mathrm{g}(2.4-2.5 \mathrm{Ghz}$ and $4.9-5.875 \mathrm{GHz})$ frequency bands are presented. Genetic Algorithm optimization (GA) is applied first, to a classical dual band printed dipole antenna schema. Later on, a pre-fractal technique is proposed on the larger strip and electromagnetic parameters are re-optimized to achieve a more compact radiator. Frequency performance of both antennas is introduced showing a VSWR $<1.5$ for a input impedance of $50 \mathrm{Ohms}$. Finally, the mean effective gain (MEG) is worked out considering several scenarios. Results for both antennas for typical indoor and outdoor environments are given using the statistical angle of arrival behavior of such environments.
\end{abstract}

Index terms - WLAN, printed dipole antennas, genetic algorithms, Mean Effective Gain.

\section{Introduction}

In the last few years, the development of wireless local area networks (WLANs) was one of the main research focus in the information and communications field. Therefore, a strong effort in antenna design to provide wireless coverage with low cost has been a key factor to accomplish the WLAN development.

In this paper, a radiating element is designed to adopt the standard printed circuit board (PCB) substrate and production technology. The uniqueness of the design comes from an evolving optimization procedure applied to a classical dual printed dipole antenna (DPDA) [1] used previously in 2 and 3G base station systems combined with a pre-fractal topology [2] to reduce the size. Additionally, since the antenna is oriented to be used in a mobile device, a traditional approach to evaluate the electromagnetic performances is not enough to predict the overall behavior in a wireless scenario. The Mean Effective Gain (MEG) [3], is a recently defined parameter to include the mobile channel characteristics (those referred to spatial and polarization properties). This parameter is computed for the radiating elements placed in typical scenarios: indoor and indoor-outdoor urban. 
In section II, the antenna geometries and design outlines are presented, showing the evolution of the GA applied. In section III, classical electromagnetic parameters (S-parameters, Gain) coming from the optimization are showed. In section IV , MEG results are presented. Finally, a conclusion is provided.

\section{Dual Printed Dipole Antenna (DPDA) Designs with GA}

\subsection{Antenna Geometries of PDA}

Fig.1 shows a schematic drawing of the antennas showing the genes involved in the genetic optimization. In the classical DPDA, two printed strip dipoles of different lengths, with the arms printed on opposite sides of an electrically thin dielectric substrate are connected through a parallel stripline (PS). In the case of the pre-fractal printed dipole antenna (PF-DPDA), the first iteration of a fractal tree is applied to the longer element so that the size can be reduced. In order to achieve an optimal dual-frequency radiator, the line polarity between the radiating elements must be inverted. The antennas were designed on a dielectric substrate of height $h=1.6 \mathrm{~mm}$, relative permittivity $\epsilon=4.5$ and loss tangent $\tan (\delta)=0.02$.
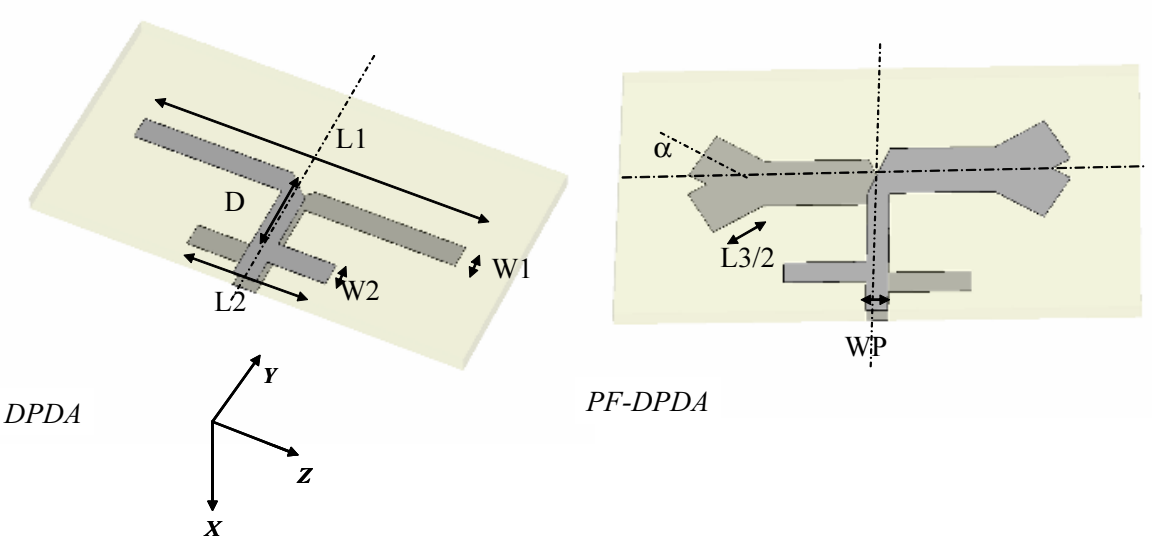

$P F-D P D A$

Fig. 1. Return Losses of DPDA and PF-DPDA

\subsection{Evolutive Optimization (GA) Results}

A genetic optimization method was applied for each geometry (DPDA PFDPDA). Six and eight genes are codified using 30 bits in a binary codification, respectively. A simple GA with typical parameters $p_{\text {cross }}=0.65, p_{\text {mut }}=0.01$, size population of 30 individuals was let to evolve during 150 generations in DPDA and 100 generations in PF-DPDA. The Fitness function was:

$$
F=\left|R_{i n}\left(\omega_{1}\right)-50\right|+\left|R_{i n}\left(\omega_{2}\right)-50\right|+\left|R_{i n}\left(\omega_{1}\right)-R_{i n}\left(\omega_{2}\right)\right|+\left|X_{i n}\left(\omega_{1}\right)\right|+\left|X_{i n}\left(\omega_{2}\right)\right|
$$


Table 1. Optimum chromosomes found by GA simple

\begin{tabular}{|c|c|c|}
\hline \multicolumn{3}{|c|}{ Gene DPDA $(\mathrm{mm})$ PF-DPDA $(\mathrm{mm})$} \\
\hline$\overline{\mathrm{L} 1}$ & 45.054 & 26.2694 \\
\hline L2 & 19.3643 & 21.3248 \\
\hline $\mathrm{D}$ & 12.5259 & 13.2189 \\
\hline W1 & 5.5733 & 5.9203 \\
\hline W2 & 2.7896 & 2.5660 \\
\hline WP & 2.9568 & 2.5019 \\
\hline L3 & - & 14.8525 \\
\hline$\alpha$ & - & 30.9282 \\
\hline
\end{tabular}

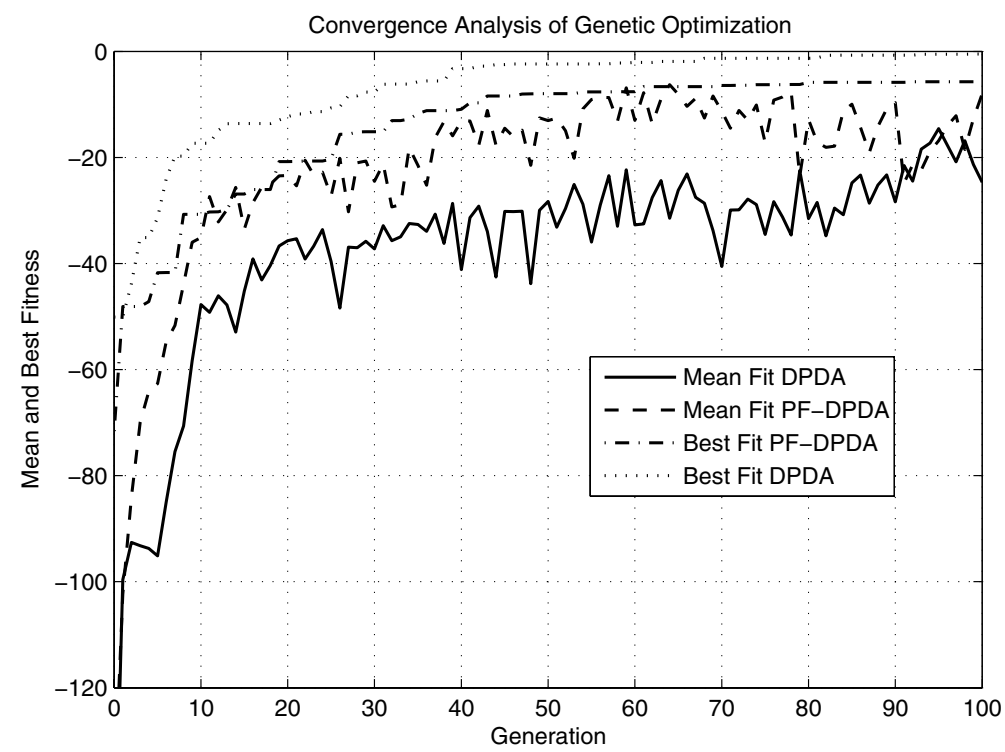

Fig. 2. Gain Pattern of PF-PDA Antenna

where $Z_{i n}\left(\omega_{i}\right)=R_{i n}\left(\omega_{i}\right)+j X_{i n}\left(\omega_{i}\right)$ is the antenna input impedance at $\omega_{i}$ frequency.

With this fitness function, a resonant $50 \Omega$ input impedance at both frequencies is looked for. The frequencies chosen for WLAN where 2.45 and $5.4 \mathrm{GHz}$. The antenna parameters were obtained from a standard MoM simulation program. Table 1 shows the optimized parameters for each antenna and Fig. 2 the fitness function convergence towards the optimum.

As observed, computing the Size Reduction as

$$
\text { size reduction }=\frac{L_{1}^{D P D A}-L_{1}^{P F D P D A}-L_{3} / 2 \cos (\alpha)}{L_{1}^{D P D A}}
$$

a $27.55 \%$ of compactness is achieved thanks to the pre-fractal method. 


\section{Classical Performances for the Radiating Configurations}

The classical analysis of antennas comprises, among others, these main quantities: the S-parameters, impedance bandwidth and the gain radiation pattern.

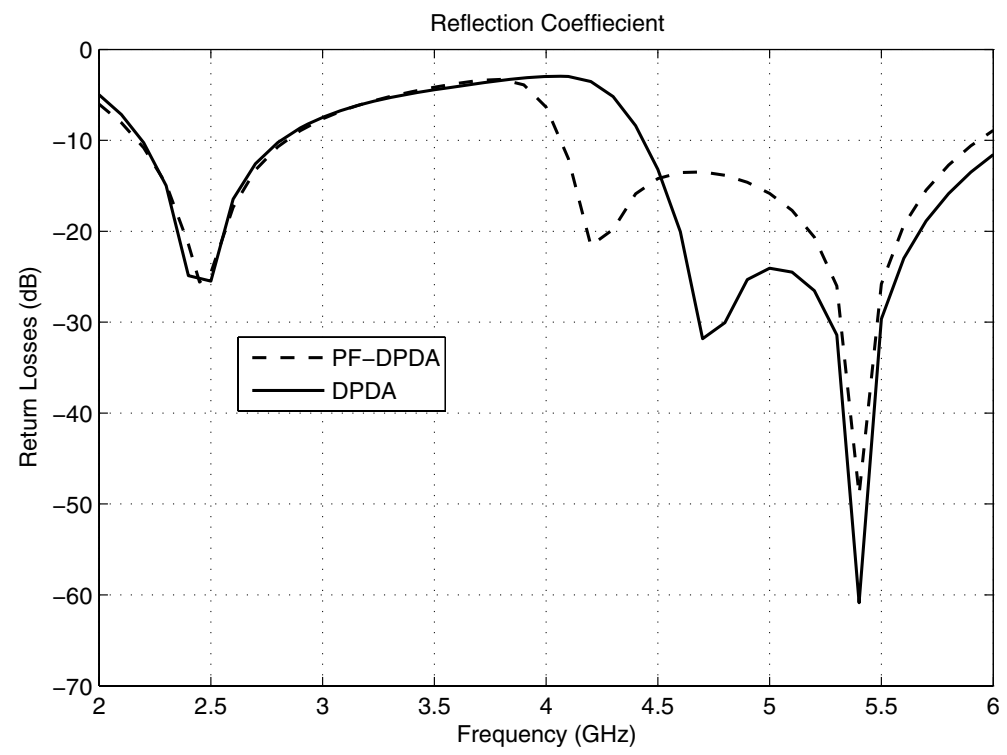

Fig. 3. Return Losses of DPDA and PF-DPDA

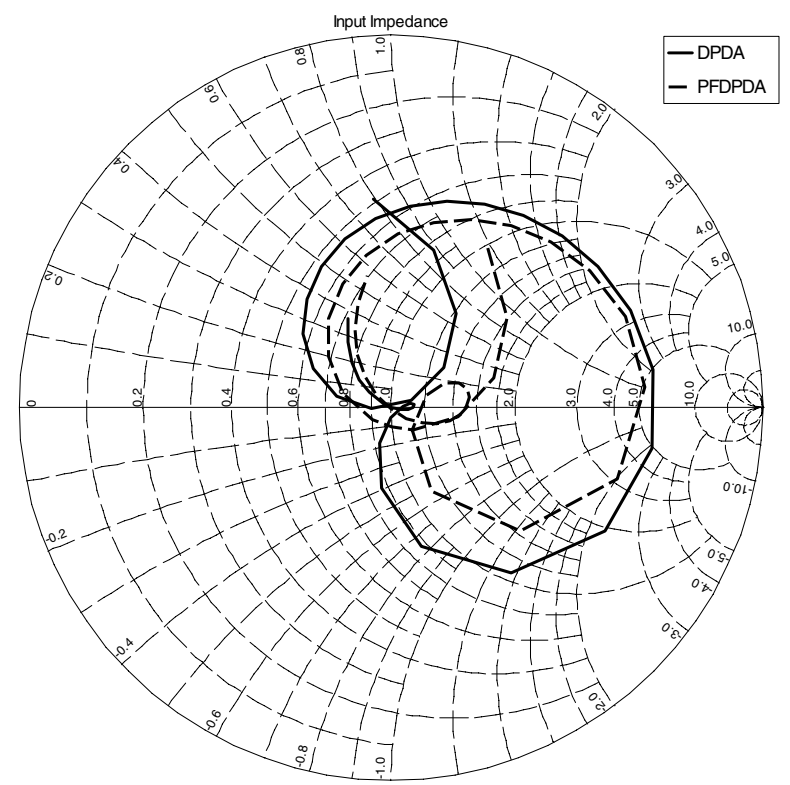

Fig. 4. Return Losses of DPDA and PF-DPDA 


\section{E-PLANE Gain}

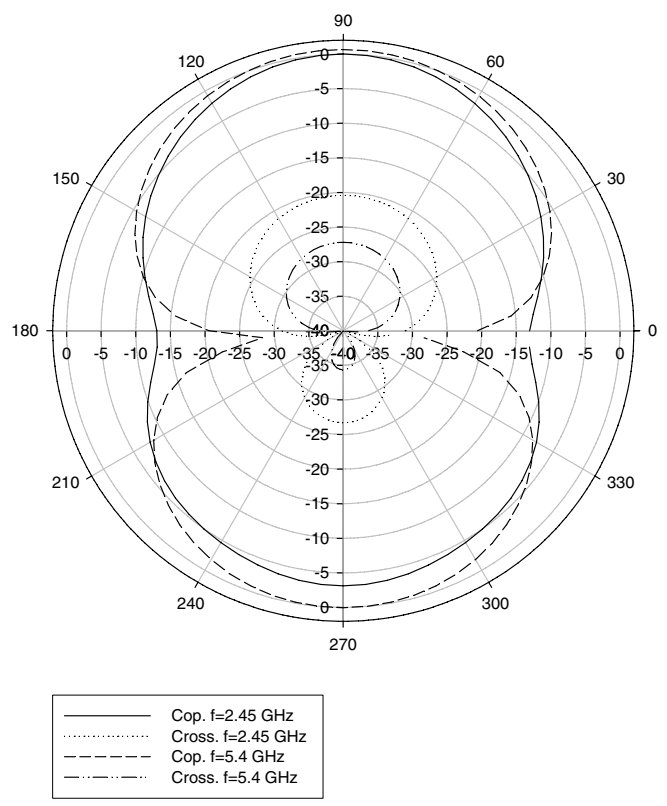

Fig. 5. Gain Pattern of PF-DPDA Antenna

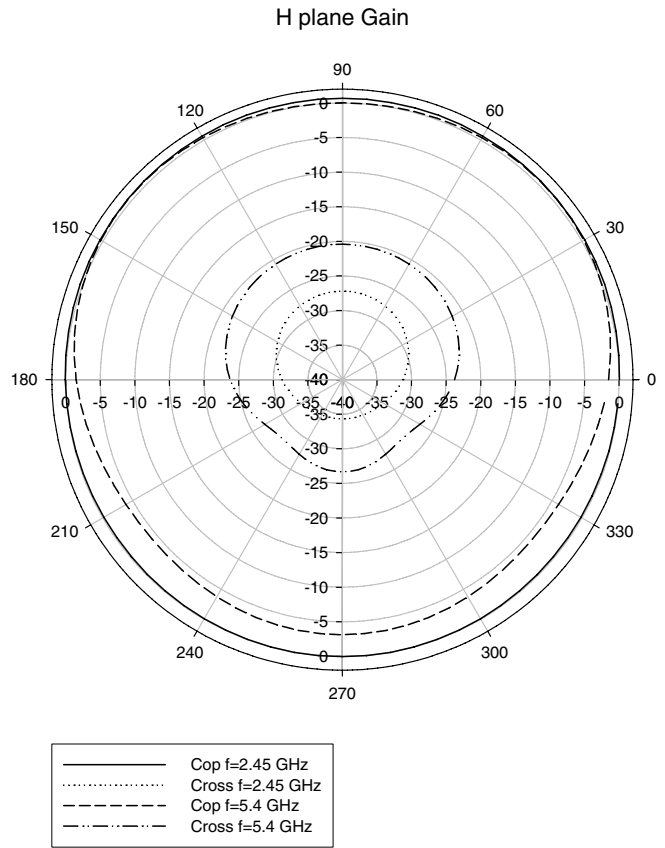

Fig. 6. Gain Pattern of PF-DPDA Antenna 
The $S_{11}$ is plotted in Fig.3-4. Considering a $|\Gamma|<-15 d B$ as bandwidth criteria, it is obvious that the antennas are radiating in the whole WLAN frequencies specified.

Regarding the pattern, Fig.5-6 represents the E and H plane cuts.It is observed that the antennas have almost an omnidirectional diagram in the lower band while in the upper band the pattern is more directive. Table 2 summarizes the classical performances.

Table 2. Antenna main traditional parameters

\begin{tabular}{lcc}
\hline Parameter & DPDA PF-DPDA \\
\hline Bandwidth WLAN 1 (MHz) & 220 & 360 \\
Bandwidth WLAN 2 (MHz) & 900 & 1455 \\
Directivity WLAN 1 (dBi) & 1.73 & 1.71 \\
Directivity WLAN 2 (dBi) & 4.6 & 3.38 \\
Gain WLAN 1 (dBi) & 0.54 & 0.67 \\
Gain WLAN 2 (dBi) & 1.11 & 0.52 \\
\hline
\end{tabular}

\section{Effective Gain Analysis in WLAN Environments}

\subsection{Method of Analysis}

As mentioned, the MEG is a statistical measurement of the antenna performance in a multipath enviroment. The mean power received from the antenna can be obtained from the radiation patterns and the statistics of the channel using this concept. The MEG of an antenna, which is defined as the ratio of the mean received to the mean incident power at the antenna, can be calculated from [4],

$$
M E G=\oint\left[\frac{\Gamma}{1+\Gamma} P_{\theta}(\Omega) G_{\theta}(\Omega)+\frac{1}{1+\Gamma} P_{\phi}(\Omega) G_{\phi}(\Omega)\right] d \Omega
$$

where $G_{\theta}$ and $G_{\phi}$ are the $\theta$ and $\phi$ polarized components of the antenna power gain pattern, $\Omega$ is the solid angle $(\theta, \phi), P_{\theta}$ and $P_{\phi}$ are the $\theta$ and $\phi$ components of the angular density functions of the incoming plane waves. $\Gamma$ is the crosspolarization power ratio, defined as the ratio of the mean received power in the vertical polarization to the mean received power in the horizontal polarization. The crosspolarization power ratio ( $\Gamma$ or also known as XPD) varies considerably, depending on the surrounding enviroment. Thus, these values must be concreted according to the mobile application of interest.

\subsection{Incident Wave Statistics for WLAN Environments}

As a result of the large amount of interest in the wireless channel, several probability density functions have been proposed [5], [6], [7], validated through measurements. First results were related to the temporal properties of the propagation environment, and finally, a focus in the angular power distribution motivated 
by the emerging MIMO systems has brought several models for the incident wave statistics. In the case of the XPR, it is shown that its value is between $0 \mathrm{~dB}$ and $9 \mathrm{~dB}$ in most cases, although in some enviroments can achieve $11 \mathrm{~dB}$.

When a WLAN indoor enviroment it is considered, two possible scenarios may be of interest:

\section{Indoor Environment}

The antenna is assumed to be working inside a building. Measurements [8] have shown that the power azimuth spectrum $P_{\phi}$ is best modeled by a Laplacian function for both polarization. A Gaussian function for the elevation is assumed. Therefore, for the DPDA and PF-DPDA antennas:

$$
\begin{aligned}
& P_{\phi}(\theta, \phi)=A_{\phi} e^{-\left|\frac{\sqrt{2} \phi}{\sigma}\right|} e^{-\left(\theta-\left[\pi / 2-m_{H}\right]\right)^{2} / 2 \sigma_{H}^{2}}, 0 \leq \theta \leq \pi, 0 \leq \phi \leq 2 \pi \\
& P_{\theta}(\theta, \phi)=A_{\theta} e^{-\left|\frac{\sqrt{2} \phi}{\sigma}\right|} e^{-\left(\theta-\left[\pi / 2-m_{V}\right]\right)^{2} / 2 \sigma_{V}^{2}}, 0 \leq \theta \leq \pi, 0 \leq \phi \leq 2 \pi
\end{aligned}
$$

For these pdfs, suitable statistic moments will be $\sigma=24^{\circ}, \sigma_{H}=9^{\circ}, \sigma_{V}=11^{\circ}$ and $m_{V}=4^{\circ}, m_{V}=2^{\circ}$. MEG will be study for XPRs between 0 and 11 , although measurements point out values around $7 \mathrm{~dB}$.

\section{Indoor-Outdoor Environment}

The antenna is assumed to be working outside a building, but close to the point access system. This corresponds to traditional gaussian pdfs in elevation and uniform distribution in azimuth. Therefore, for the DPDA and PF-DPDA antennas:

$$
\begin{aligned}
& P_{\phi}(\theta, \phi)=A_{\phi} e^{-\left(\theta-\left[\pi / 2-m_{H}\right]\right)^{2} / 2 \sigma_{H}^{2}}, 0 \leq \theta \leq \pi, 0 \leq \phi \leq 2 \pi \\
& P_{\theta}(\theta, \phi)=A_{\theta} e^{-\left(\theta-\left[\pi / 2-m_{V}\right]\right)^{2} / 2 \sigma_{V}^{2}}, 0 \leq \theta \leq \pi, 0 \leq \phi \leq 2 \pi
\end{aligned}
$$

In both cases, $A_{\theta}$ and $A_{\phi}$ are constants that must fulfill:

$$
\int_{0}^{2 \pi} \int_{0}^{\pi} P_{\theta}(\theta, \phi) \sin \theta d \theta d \phi=\int_{0}^{2 \pi} \int_{0}^{\pi} P_{\phi}(\theta, \phi) \sin \theta d \theta d \phi=1
$$

For these pdfs, suitable statistic moments will be $\sigma_{H}=8^{\circ}, \sigma_{V}=15^{\circ}$ and $m_{V}=1^{\circ}, m_{V}=2^{\circ}$. MEG will be study for XPRs between 0 and 11 , although measurements point out values around $11 \mathrm{~dB}$.

\subsection{Results}

Fig. 7 shows results for MEG in indoor-outdoor environment, for both frequency bands. As expected, if XPR increases, the MEG is improving approaching to theoretical Gain.

As seen, the antenna performance is worse at the higher frequency than at lower,and the antennas have almost the same MEG,with slightly differences. Fig 8 presents the results for the PF-DPDA in both enviroments. It is revealed that, in the case of the indoor environment, the MEG is slightly worse. As the Laplacian distribution is sharper than the uniform in the center, the indoor MEG is lower compared to the outdoor MEG. 


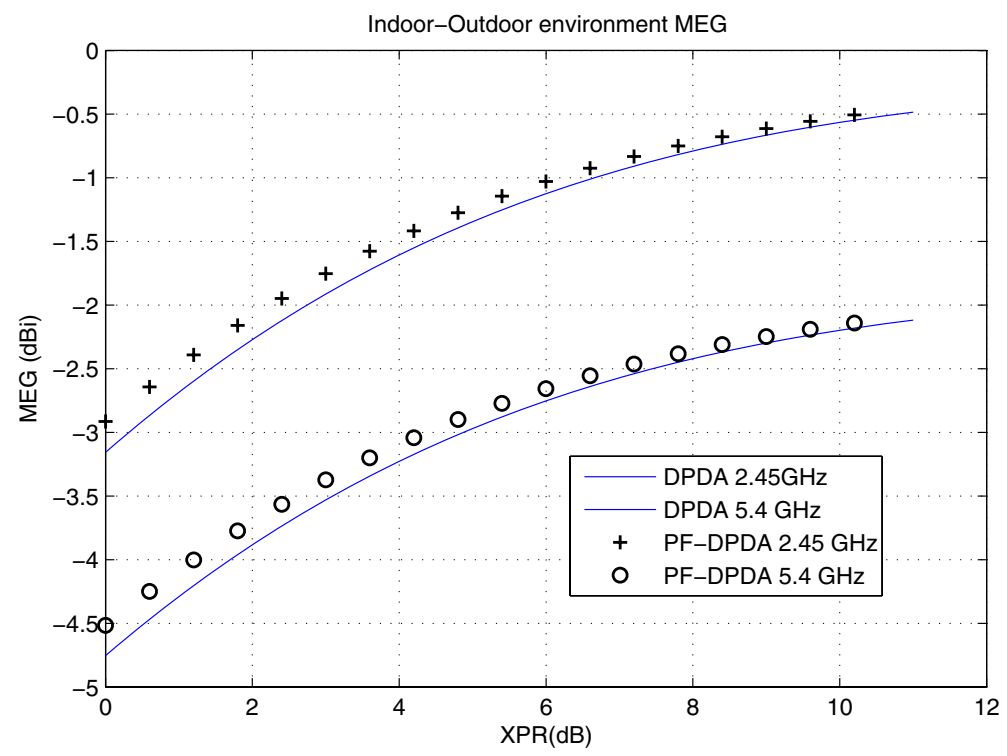

Fig. 7. MEG in indoor-outdoor environment

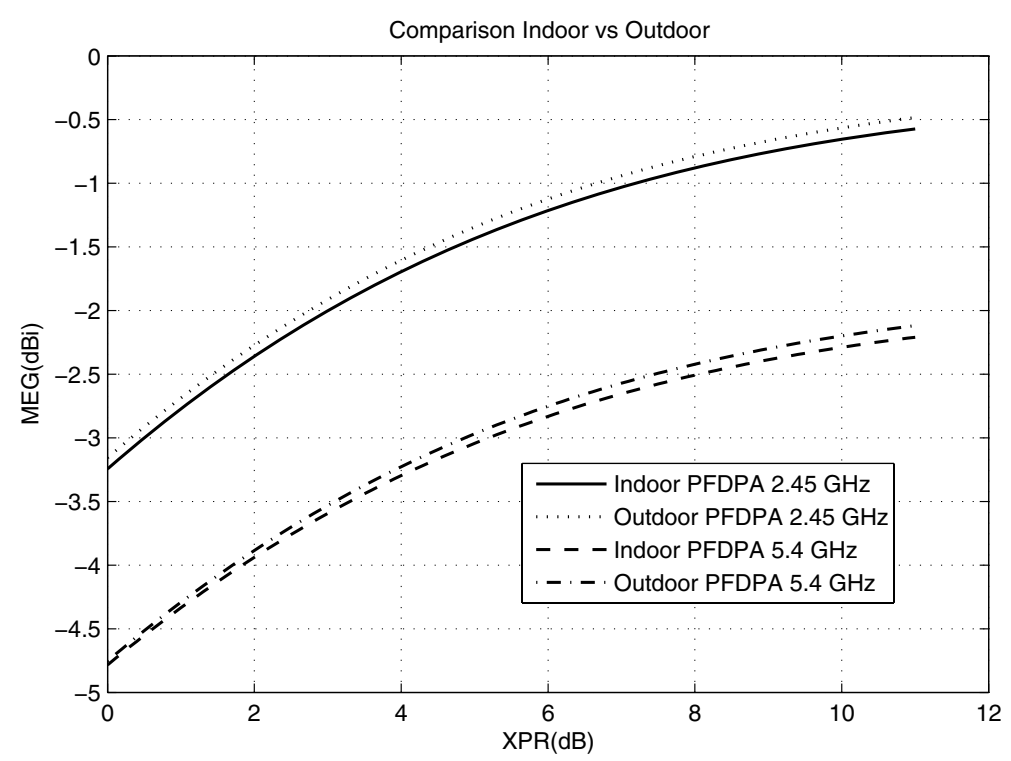

Fig. 8. MEG in indoor-outdoor environment

\section{Conclusion}

This paper shows a novel genetically pre-fractal printed dipole antenna for WLAN frequency bands. The antenna is analyzed in classical terms showing good performances in both bands. Additionally, the Mean Effective Gain is 
obtained two typical scenarios, revealing an identical performance in the compacted antenna and the standard.

\section{Acknowledgement}

This work has been funded by the Ministry of Education and Science and the European funds of regional development (FEDER) under the project TEC 200404529/TCM and the European Union through the Program Marco under the project PULSERS PHASE-2 (Pervasive Ultra-wideband Low Spectral Energy Radio Systems PHASE 2).

\section{References}

[1] F. Tefiku and C. A. Grimes, Design of Broad-Band and Dual Band Antennas Comprised of Series-Fed Printed-Strip Dipole Pairs, IEEE Trans. Antennas and Propagat. Vol.48,No 6, June, 2000.

[2] J.S. Petko and D. H. Werner, Miniature reconfigurable three-dimensional fractal tree antennas, IEEE Trans. Antennas and Propagat. Vol.52,No 8, Aug, 2004.

[3] de Mingo, J.; Valdovinos, A.; Gutierrez, F.; Gonzalez, J.M., Inverted-F antenna with parasitic elements for TETRA handset, Vehicular Technology Conference, 1999. VTC 1999 - Fall. IEEE VTS 50th Volume 3, 19-22 Sept. 1999

[4] Douglas,M.G.; Okoniewski, M.; Stuchly, M.A, A planar diversity antenna for handheld PCS devices, Vehicular Technology, IEEE Transactions on Volume 47, Issue 3, Aug. 1998

[5] Kalliola, K.; Sulonen, K.; Laitinen, H.; Kivekas, O.; Krogerus, J.; Vainikainen, P., Angular power distribution and mean effective gain of mobile antenna in different propagation environments, Vehicular Technology, IEEE Transactions on Volume 51, Issue 5, Sept. 2002

[6] Waldschmidt, C.; Wiesbeck, W., Compact wide-band multimode antennas for MIMO and diversity,Antennas and Propagation, IEEE Transactions on Volume 52, Issue 8, Aug. 2004

[7] Hajian, M.; Nikookar, H.; der Zwan, Fv.; Ligthart, L.P., Branch correlation measurements and analysis in an indoor Rayleigh fading channel for polarization diversity using a dual polarized patch antenna,Microwave and Wireless Components Letters, IEEE Volume 15, Issue 9, Sept. 2005

[8] pencer, Q.H.; Jeffs, B.D.; Jensen, M.A.; Swindlehurst, A.L., Modeling the statistical time and angle of arrival characteristics of an indoor multipath channel,Selected Areas in Communications, IEEE Journal on Volume 18, Issue 3, March 2000 